\title{
Electron and hole drift velocity in chemical vapor deposition diamond
}

\author{
Markus Gabrysch, ${ }^{1}$ Saman Majdi, ${ }^{1}$ Daniel J. Twitchen, ${ }^{2}$ and Jan Isberg ${ }^{1, a)}$ \\ ${ }^{1}$ Division for Electricity, Uppsala University, Box 534, S-751 21 Uppsala, Sweden \\ ${ }^{2}$ Element Six Ltd, King's Ride Park, Ascot, Berkshire, SL5 8BP, United Kingdom
}

(Received 20 August 2010; accepted 15 January 2011; published online 24 March 2011; publisher error corrected 05 April 2011)

\begin{abstract}
The time-of-flight technique has been used to measure the drift velocities for electrons and holes in high-purity single-crystalline CVD diamond. Measurements were made in the temperature interval $83 \leq T \leq 460 \mathrm{~K}$ and for electric fields between 90 and $4 \times 10^{3} \mathrm{~V} / \mathrm{cm}$, applied in the $\langle 100\rangle$ crystallographic direction. The study includes low-field drift mobilities and is performed in the low-injection regime to perturb the applied electric field only minimally. (c) 2011 American Institute of Physics. [doi:10.1063/1.3554721]
\end{abstract}

\section{INTRODUCTION}

Diamond is a wide bandgap semiconductor with many superior material properties such as high carrier mobilities, high saturation velocity, high breakdown field, and highest thermal conductivity of all materials. These extreme properties make single-crystalline epitaxially grown (SC-CVD) diamond an outstanding candidate for many electronic device and detector applications where high-power, high-frequency, ultra-fast response time or radiation hardness are crucial. Doping diamond, however, is still a challenge. Diamond lacks a shallow dopant that is fully thermally activated at room temperature. Therefore, the more promising device concepts contain thin delta-doped layers with a very high dopant concentration, above the Mott transition, that are fully activated in conjunction with undoped (intrinsic) layers where charges are transported. This is one reason why an improved understanding of transport in high-quality undoped layers with high carrier mobilities is important.

Mobilities of charge carriers in semiconductors are usually measured using the Hall effect. This method can not be applied in the case of insulating intrinsic diamond. Instead, the time-of-flight (ToF) method, also often referred to as transient current technique (TCT), can be applied. In this case, electronholes pairs can be created by $\alpha$-particles, ${ }^{1-3} \beta$-particles, ${ }^{4}$ pulsed electron beams, ${ }^{5}$ pulsed x-rays ${ }^{6,7}$ or a pulsed UV laser. $^{8-11}$ The motion of the free charge carriers in an applied electric field induces a current which is measured.

In the 1980s, detailed studies for electron and hole drift velocities and mobilities for natural diamond were performed by the group of Nava, Canali, Reggiani et al. ${ }^{12-14}$ in the temperature range of $85-700 \mathrm{~K}$ with electric fields up to $60 \mathrm{kV} / \mathrm{cm}$. More recently, several studies of drift velocity measurements in single-crystalline diamond have been performed at room temperature. ${ }^{1-3}$

In this paper, we present a systematic set of experimental data for intrinsic SC-CVD diamond of both hole and electron drift velocities in the temperature range between 83 and $460 \mathrm{~K}$. The electric fields range between 90 and $4 \times 10^{3}$

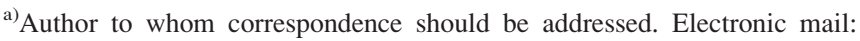
jan.isberg@angstrom.uu.se.
}

$\mathrm{V} / \mathrm{cm}$ and were applied in the $\langle 100\rangle$ direction of the singlecrystalline samples.

\section{EXPERIMENTAL TECHNIQUE}

The presented carrier drift velocities were obtained through the time-of-flight technique. Free charge carriers are generated by short ( 3 ns FWHM) UV pulses from a quintupled Nd-YAG laser with $10 \mathrm{~Hz}$ repetition frequency and $213 \mathrm{~nm}$ wavelength, which corresponds to a photon energy just above the bandgap of diamond $(5.47 \mathrm{eV})$. Several interference filters and neutral density filters block lower harmonics and allow for reducing the intensity to the desired magnitude (see Fig. 1). A semitransparent Ti/Al or Ni mesh contact makes it possible to apply both a relatively homogenous electric field and to create electron-hole pairs within the vicinity (a few micrometers) of the illuminated side of the sample due to the strong absorption process of the UV photons in diamond.

The polarity of the applied bias voltage determines the type of carrier that drifts through the bulk of the sample to the Ti/Al or Ni backside contact. The charge accumulation can be kept to a minimum by pulsing the bias (50 $\mu$ s/pulse).

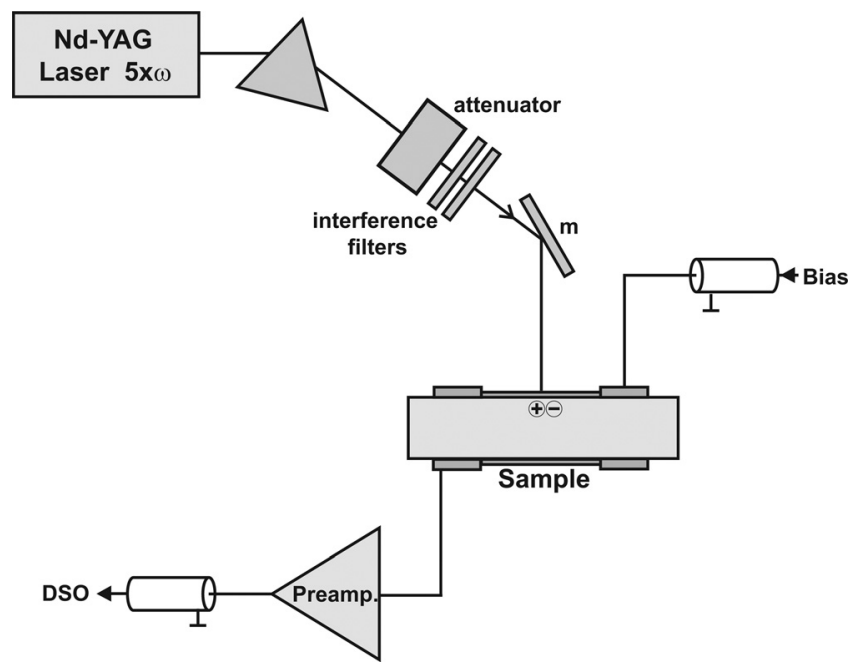

FIG. 1. Schematic of the ToF setup. The sample is illuminated with $3 \mathrm{~ns}$ (FWHM) 213 nm UV light from a quintupled Nd-YAG laser. 
TABLE I. Overview of the three samples selected for this study.

\begin{tabular}{llc}
\hline \hline & Front contact - back contact & Thickness $(\mu \mathrm{m})$ \\
\hline Sample 1 & $\mathrm{Ti} / \mathrm{Al}$ - Ti/Al & 490 \\
Sample 2 & $\mathrm{Ni}$ - Ni & 510 \\
Sample 3 & $\mathrm{Ti} / \mathrm{Al}$ - Ti/Al & 690 \\
\hline
\end{tabular}

The middle column shows the different metallization used for the front side (mesh) contact and back side (solid) contact.
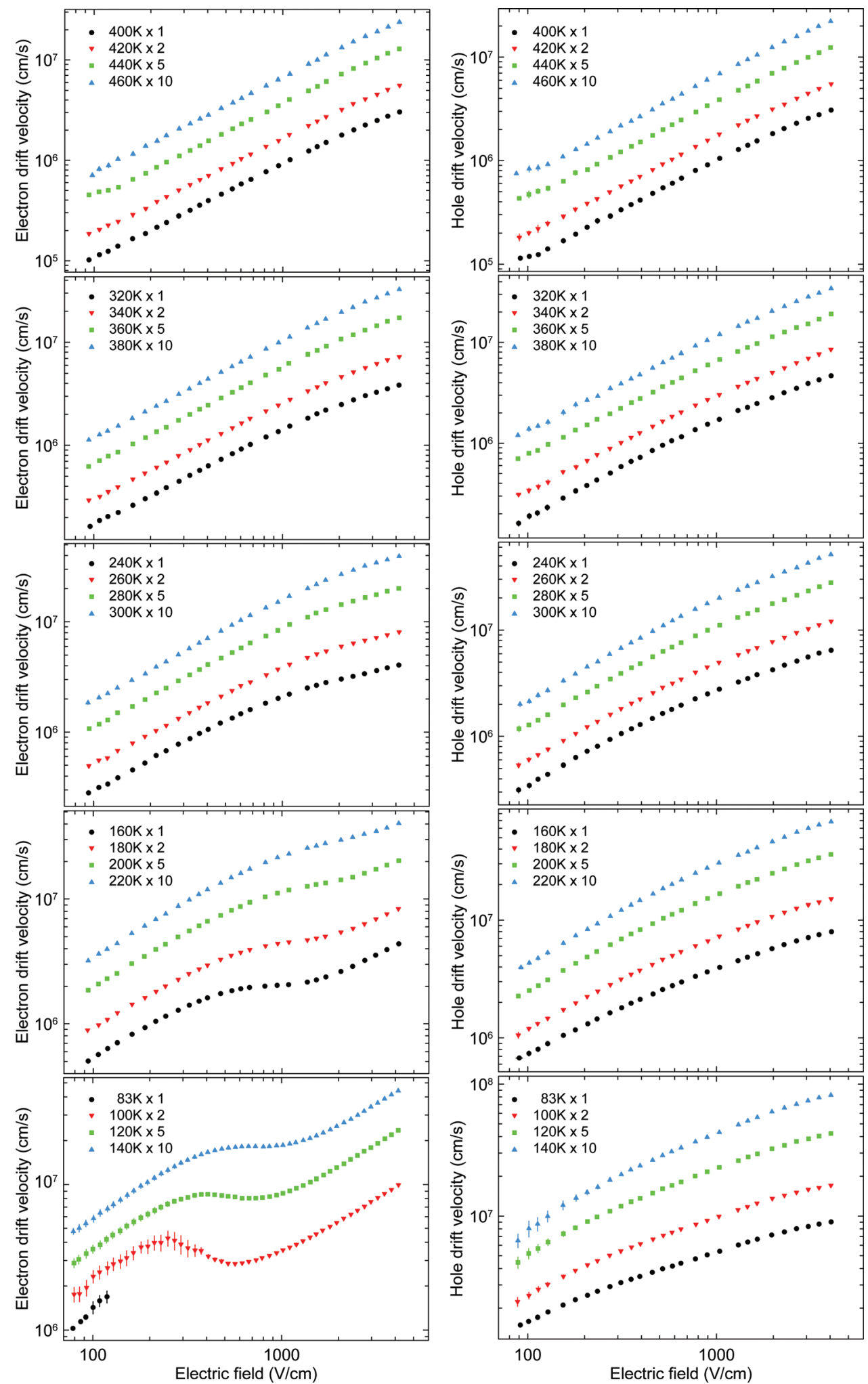

The current is amplified and stored in a fast digital oscilloscope (Tektronics TDS $684 \mathrm{C}, 5 \mathrm{Gs} / \mathrm{s}$ ) that is triggered by the Q-switch signal of the laser.

The transit time $\tau$ of the charge carriers can be easily determined from the time difference between the rising and falling edge of the current pulses. The drift velocity $v_{d}$ is then simply the quotient of sample thickness $d$ and $\tau$, assuming that the charge is created close to the front contact and in the center of the mesh.
FIG. 2. (Color online) Drift velocities for electrons and holes in the temperature range $83-460 \mathrm{~K}$. 
The samples were mounted in vacuum in a liquid nitrogen cooled cryostat. The sample temperature was monitored by a calibrated silicon diode (DT-670) and a temperature controller (Lake Shore 331). The temperature interval for this study was fixed by the temperature of liquid nitrogen and the upper temperature limit of the cryostat $(470 \mathrm{~K})$.

\section{SAMPLES}

The three single-crystalline CVD diamond plates selected for this study (see Table I) were produced and supplied by Element Six Ltd. The material was deposited homoepitaxially on specially prepared HPHT synthetic diamond substrates in a microwave plasma-assisted CVD reactor. After synthesis, the epitaxial overlayers (490, 510, and $690 \mu \mathrm{m}$ in thickness) were separated from their HPHT diamond substrate by a laser cutting technique and polished to give freestanding high purity plates with nitrogen impurities below $5 \times 10^{14} \mathrm{~cm}^{-3}$. Both sides of the samples were metallized by sputtering, either with $\mathrm{Ti} / \mathrm{Al}$ or Ni in a physical vapor deposition (PVD) system. The front contacts were patterned by means of standard optical lithography techniques to form semitransparent mesh patterns, $4 \mathrm{~mm}$ in diameter, on the $\{100\}$ surfaces.

\section{RESULTS}

The measured drift velocities for electrons and holes are shown in Fig. 2. (Note that the curves are multiplied by factors 2, 5, and 10 to obtain offsets.) The data points were obtained in the following way. For fixed temperature and electric field, the current transit was recorded for each sample as an average over 50 laser shots. From the shape of the current traces (nearideal rectangular pulses, even for very low bias voltages), it could be seen that polarization effects were very small. The time-of-flight $\tau$ is the full-width half-maximum (FWHM) of the current transit, and its error was estimated from the corresponding full-width times for $(50 \pm 5) \%$ of the maximum. Then a (weighted) least-square fit to the results from the different samples was applied yielding a (weighted) mean value with error bounds for the drift velocities.

The drift mobility data shown in Fig. 3 are based on fitting the data to the semiempirical relation

$$
v_{d}=\frac{\mu E}{1+\mu E / v_{\text {sat }}},
$$

which relates the carrier drift velocity $v_{d}$ to the saturation velocity $v_{\text {sat }}$ and the applied electric field $E$. This equation can be rewritten simply by using $v=d / \tau$ and $E=U / d$ to rewrite Eq. (1) as

$$
\frac{1}{v_{d}}=\frac{\tau}{d}=\frac{1}{v_{\text {sat }}}+\frac{1}{\mu} \cdot \frac{1}{E}
$$

Then for each temperature, the part of the data that was consistent with Eq. (1), that is, that showed linearity when plotting drift velocity $v_{d}$ versus reciprocal electric field $1 / E$, was fitted by applying a (weighted) least-square fit to Eq. (2) for all three samples simultaneously. Particularly for temperatures below $200 \mathrm{~K}$, only data with $|E| \leq 400 \mathrm{~V} / \mathrm{cm}$ were taken into

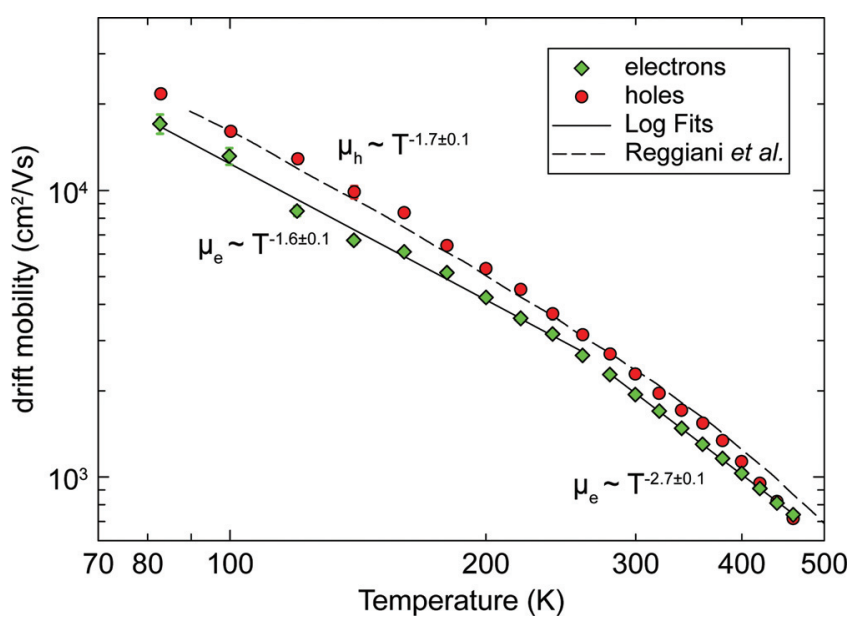

FIG. 3. (Color online) Low field drift mobilities for electrons (circles) and holes (squares) in the temperature range $83-460 \mathrm{~K}$. Data are based on (weighted) least-square fits. The dashed line was reprinted with permission from Reggiani et al., Phys. Rev. B 23, 3050 (1981). (Copyright 1981 by the American Physical Society.)

account. The mobility is simply the inverse slope of the fit and the saturation velocity is the $y$-axis intercept.

\section{DISCUSSION AND CONCLUSION}

The measured drift velocities show very little spread across the different samples studied in this paper; this indicates that the quality of the SC-CVD diamond layers has come to stage where it is possible to talk not only about the properties of the specific sample, but also about the material's electronic properties. The choice of contact metal-Ti/ $\mathrm{Al}$ or $\mathrm{Ni}$ - did not seem to have an impact on the results.

Figure 3 shows that for both electrons and holes, the mobility follows the typical $T^{-3 / 2}$ dependence for $T<280 \mathrm{~K}$, which is an indication that acoustic phonon scattering is the limiting mechanism in that temperature range. For higher temperatures, the slope becomes steeper indicating the onset of intervalley phonon scattering. This is in good agreement with previous findings; see for example, Refs. 9 and 12-14.

The local maximum for electron velocities at low temperatures $(100-140 \mathrm{~K})$ is believed to be due to field-dependent conduction valley repopulation. We intend to investigate this phenomenon in more detail in the future and compare with Monte Carlo simulations.

The hole drift velocities are in good agreement with the data for ultra-pure natural diamond in Reggiani et al. ${ }^{14}$ In that paper, only drift velocities exceeding $10^{6} \mathrm{~cm} / \mathrm{s}$ for nine temperatures in the range $85-700 \mathrm{~K}$ are presented. The hole drift mobilities agree for temperatures down to $300 \mathrm{~K}$. For lower temperatures, the values presented in this paper are around 50\% higher than the experimental results of Reggiani et al., but they agree very well with their theoretical calculations (see Fig. 5 in Reggiani et al. ${ }^{14}$ ), which are based on a two-band model (heavy and light holes) with spherical and parabolic bands. Only lattice scattering mechanisms (acoustic and nonpolar scattering effects) are taken into account.

Our results for electron drift velocities were compared to the data for 85 and $300 \mathrm{~K}$ presented by Nava et al. and showed the same behavior for those temperatures even though 
our results for both the electron drift velocities and mobilities were slightly lower. ${ }^{13}$ Compared to the high injection regime, ${ }^{9}$ we observe lower mobilities in our case of low injection.

\section{ACKNOWLEDGMENTS}

M.G., S.M., and J.I. would like to thank the Swedish Research Council (VR) and the Foundation for Strategic Environmental Research (MISTRA) for financial support.

${ }^{1}$ H. Pernegger, S. Roe, P. Weilhammer, V. Eremin, H. Frais-Kolbl, E. Griesmayer, H. Ka-gan, S. Schnetzer, R. Stone, W. Trischuk, D. Twitchen, and A. Whitehead, J. Appl. Phys. 97, 073704 (2005).

${ }^{2}$ M. Pomorski, E. Berdermann, M. Ciobanu, A. Martemyianov, P. Moritz, M. Rebisz, and B. Marczewska, Phys. Status Solidi A 202, 2199 (2005).

${ }^{3}$ M. Pomorski, E. Berdermann, A. Caragheorgheopol, M. Ciobanu, M. Kiscaron, A. Marte-myianov, C. Nebel, and P. Moritz, Phys. Status Solidi A 203, 3152 (2006).

${ }^{4}$ C. Canali, E. Gatti, S. Kozlov, P. Manfredi, C. Manfredotti, F. Nava, and A. Quirini, Nucl. Instrum. Methods. 160, 73 (1979).

${ }^{5}$ A. A. Quaranta, C. Canali, and G. Ottaviani, Rev. Sci. Instrum. 41, 1205 (1970).
${ }^{6}$ L. S. Pan, S. Han, D. R. Kania, S. Zhao, K. K. Gan, H. Kagan, R. Kass, R. Malchow, F. Morrow, W. F. Palmer, C. White, S. K. Kim, F. Sannes, S. Schnetzer, R. Stone, G. B. Thomson, Y. Sugimoto, A. Fry, S. Kanda, S. Olsen, M. Franklin, J. W. Ager III, and P. Pianetta, J. Appl. Phys. 74, 1086 (1993).

${ }^{7}$ M. Gabrysch, E. Marklund, J. Hajdu, D. J. Twitchen, J. Rudati, A. M. Lindenberg, C. Caleman, R. W. Falcone, T. Tschentscher, K. Moffat, P. H. Bucksbaum, J. Als-Nielsen, A. J. Nelson, D. P. Siddons, P. J. Emma, P. Krejcik, H. Schlarb, J. Arthur, S. Brennan, J. Hastings, and J. Isberg, J. Appl. Phys. 103, 064909 (2008).

${ }^{8}$ C. E. Nebel, J. Münz, M. Stutzmann, R. Zachai, and H. Güttler, Phys. Rev. B 55, 9786 (1997).

${ }^{9}$ J. Isberg, J. Hammersberg, E. Johansson, T. Wikstrom, D. J. Twitchen, A. J. Whitehead, S. E. Coe, and G. A. Scarsbrook, Science 297, 1670 (2002).

${ }^{10}$ J. Isberg, M. Gabrysch, A. Tajani, and D. J. Twitchen, Semicond. Sci. Technol. 21, 1193 (2006)

${ }^{11}$ M. Nesladek, A. Bogdan, W. Deferme, N. Tranchant, and P. Bergonzo, Diamond Relat. Mater. 17, 1235 (2008).

${ }^{12}$ F. Nava, C. Canali, M. Artuso, E. Gatti, P. F. Manfredi, and S. F. Kozlov, IEEE Trans. Nucl. Sci. 26, 308 (1979).

${ }^{13}$ F. Nava, C. Canali, C. Jacoboni, L. Reggiani, and S. F. Kozlov, Solid State Commun. 33, 475 (1980).

${ }^{14}$ L. Reggiani, S. Bosi, C. Canali, F. Nava, and S. F. Kozlov, Phys. Rev. B 23, 3050 (1981) 